\title{
Alterações no vocabulário expressivo de crianças com desvio fonológico
}

\section{Expressive vocabulary deficits of children with phonological disorders}

\author{
Helena Bolli Mota ${ }^{1}$, Tassiana Isabel Kaminski² ${ }^{2}$ Mirele Rezendes Flôres Nepomuceno ${ }^{3}$, Márcia de Lima Athayde
}

RESUMO

\begin{abstract}
Objetivo: Verificar os processos de substituição mais frequentes e os campos conceituais mais alterados em uma prova de vocabulário expressivo de crianças com desvio fonológico, relacionando-os com a gravidade do desvio fonológico destas crianças. Métodos: A amostra foi composta por 44 sujeitos com desvio fonológico, com idades entre três anos e cinco meses e oito anos e seis meses. Neste estudo, foram utilizados dados extraídos de bancos de dados de projetos de pesquisa em andamento no Centro de Estudos de Linguagem e Fala da Universidade Federal de Santa Maria. A amostra foi dividida em quatro grupos (grave, moderado-grave, leve-moderado e leve), de acordo com a gravidade do desvio fonológico, determinada através do cálculo do Percentual de Consoantes Corretas. Foi aplicado o teste de linguagem infantil ABFW - Vocabulário. Após, realizou-se análise de correlação entre os processos de substituição e os campos conceituais com a gravidade do desvio fonológico. Resultados: O processo de substituição "co-hipônimo" é o mais utilizado pelas crianças, independente do grau de gravidade. Observou-se também que crianças pertencentes ao grau médio-moderado realizam maior quantidade relevante de processos de substituição. No campo conceitual "locais" encontrou-se maior número de crianças com alteração, sendo que o grau médio-moderado foi o que mais apresentou campos conceituais alterados. Conclusões: O grau médio-moderado é o que mais apresenta dificuldade em prova de vocabulário expressivo, o processo de substituição mais realizado é o uso de co-hipônimo e o campo conceitual "locais" é o que mais apresenta alteração pelas crianças de um modo geral.
\end{abstract}

Descritores: Vocabulário; Testes de linguagem; Distúrbios da fala; Criança; Fala; Linguagem infantil

\section{INTRODUÇÃO}

A linguagem pode ser compreendida como uma função mental altamente complexa, sendo constituída por aspectos linguísticos e pela interligação de seus aspectos sintáticos, semânticos, morfológicos e pragmáticos, permite que a comunicação ocorra ${ }^{(1)}$.

Embora o relacionamento entre fonologia e semântica não seja tão aparente porque a fonologia lida com um aspecto estrutural da língua enquanto que a semântica é, geralmente, considerada como um aspecto conceitual, muitas investigações

Trabalho realizado no Centro de Estudos de Linguagem e Fala da Universidade Federal de Santa Maria - UFSM - Santa Maria (RS), Brasil, com fomento do Conselho Nacional de Desenvolvimento Científico e Tecnológico - CNPq por meio de bolsas de iniciação científica concedidas à segunda e à terceira autoras.

(1) Doutora; Professora do Curso de Fonoaudiologia e do Curso de Mestrado em Distúrbios da Comunicação Humana da Universidade Federal de Santa Maria - UFSM - Santa Maria (RS), Brasil.

(2) Acadêmica do Curso de Fonoaudiologia da Universidade Federal de Santa Maria - UFSM - Santa Maria (RS), Brasil.

(3) Acadêmica do Curso de Fonoaudiologia da Universidade Federal de Santa Maria - UFSM - Santa Maria (RS), Brasil.

(4) Pós-graduanda em Distúrbios da Comunicação Humana pela Universidade Federal de Santa Maria - UFSM - Santa Maria (RS), Brasil.

Endereço para correspondência: Tassiana Isabel Kaminski. R. Clemente Soltis, 067, Guarani das Missões - RS, CEP: 97950-000. E-mail: tassikaminski@yahoo.com.br

Recebido em: 7/1/2008; Aceito em: 20/10/2008 têm mostrado que certos fatores semânticos influenciam a precisão fonética $^{(2)}$.

O processo de aquisição e desenvolvimento fonológico ocorre de maneira gradual, até que haja o estabelecimento do sistema fonológico, de acordo com a comunidade linguística que a criança está inserida. A idade esperada para o estabelecimento deste sistema fonológico é até os cinco anos de idade, podendo estender-se dos quatro até, no máximo, os seis anos de idade. Porém, para algumas crianças, o processamento das informações fonológicas acontece de maneira diferente do esperado. Estas crianças têm dificuldade na organização mental dos sons da língua, no estabelecimento do sistema fonológico alvo, bem como na adequação do input recebido. Estes casos são denominados desvios fonológicos ${ }^{(3)}$.

Algumas crianças com idades superiores a quatro anos apresentam alteração no desenvolvimento normal da fala, tornando-a, em alguns casos, ininteligível. Trata-se de crianças que apresentam desvio fonológico, o qual está além de apenas uma dificuldade articulatória, pois se trata de uma alteração na organização do sistema fonológico ${ }^{(4)}$.

Embora sua causa ainda não esteja claramente definida, esta desordem caracteriza-se por alterações na produção dos sons e uso inadequado das regras fonológicas da língua ${ }^{(5)}$. Alguns autores observaram que a maior dificuldade para os sujeitos com desvio fonológico está em compreender e/ou usar as regras fonológicas, e não, necessariamente, na produção dos sons, na medida em que às vezes um som é produzido de 
forma correta e às vezes não ${ }^{(6)}$. Portanto, devemos considerar as evidências de que a dificuldade destas crianças está na organização linguística-cognitiva do sistema fonológico da língua ${ }^{(7)}$.

Pode-se verificar em crianças com desvio fonológico um desenvolvimento atrasado, variável e diferente quando comparadas ao de crianças com aquisição normal da fonologia. Muitas destas crianças apresentam ainda prejuízo em outras áreas da linguagem, tais como sintaxe, morfologia e léxico ${ }^{(8)}$.

Em estudo que relacionou o grau de gravidade do desvio fonológico com a idade cronológica, concluiu-se que diferentes graus de desvio podem ocorrer em diferentes idades, ou seja, são independentes ${ }^{(3)}$.

Aspectos pragmáticos, semânticos, morfossintáticos e fonológicos não podem ser separados, pois agem de forma conjunta no desenvolvimento das habilidades lingüísticas*. Levando-se em consideração esta afirmação de Reed, citada por Befi-Lopes e Gandara ${ }^{(9)}$, não é possível descartar a relação existente entre o desenvolvimento da fonologia e do léxico, na qual um pode interferir no desempenho do outro.

As crianças passam por diversas fases no seu desenvolvimento, as quais contribuem de maneiras diferentes para a aquisição do léxico e da linguagem: simultaneamente, ocorre a aquisição do significado das palavras, a sua produção, bem como o uso funcional destas ${ }^{(10)}$. Durante a evolução da linguagem, o desenvolvimento do léxico e da fonologia está interligado, mesmo que existam variações individuais ${ }^{(11)}$.

O desvio fonológico tem aparente desordem no nível da fonologia, tratando-se de uma patologia da linguagem ${ }^{(8)}$. O desvio fonológico envolve um déficit na aquisição fonológica, podendo ocorrer dificuldades em vários níveis: discriminação de diferenças fonéticas, reconhecimento dos contrastes fonológicos e representação destes contrastes no léxico, modificações dos sons da fala pelo uso de regras fonológicas da criança e imprecisão articulatória ${ }^{(12)}$.

Os erros fonológicos expressam a desorganização do sistema de som, e que esta esteja relacionada às alterações na representação fonológica do item lexical, o que poderia contribuir para a lentidão no acesso das representações lexicais nas crianças com desvios fonológicos ${ }^{(13)}$.

O desenvolvimento fonêmico normal é finalizado aos sete anos de idade, enquanto que o desenvolvimento semântico é um processo longo, que se desenvolve durante toda a vida. Assim, a linguagem se divide em subsistemas que são adquiridos em uma seqüência postero-anterior, começando com os aspectos semântico e textual, que são os mais precoces, seguidos pelos aspectos fonético-fonológicos, os aspectos gramaticais, e terminando com a pragmática. Todos esses aspectos estão estreitamente interligados no desenvolvimento da linguagem ${ }^{(14)}$. O período entre dois e quatro anos de idade corresponde ao desenvolvimento da capacidade de nomeação, através do léxico de verbos ${ }^{(15)}$.

O objetivo desse trabalho foi verificar, em crianças com desvio fonológico, os tipos de processos de substituição mais frequientes e os campos conceituais mais alterados em uma prova de vocabulário expressivo relacionando-os com a gravidade do desvio fonológico.

\section{MÉTODOS}

Esta pesquisa foi realizada a partir de dados clínicos de crianças participantes do projeto de pesquisa "Vocabulário expressivo e habilidades de memória de trabalho em crianças com desenvolvimento fonológico normal e desviante" aprovado pelo Comitê de Ética em Pesquisa do Centro de Ciências da Saúde da Universidade Federal de Santa Maria sob número 0102.0.243.000-07.

O referido projeto encontra-se em andamento no Centro de Estudos de Linguagem e Fala (CELF) do Serviço de Atendimento Fonoaudiológico (SAF) da UFSM.

Os responsáveis pelos sujeitos envolvidos no estudo leram e assinaram o Termo de Consentimento Livre e Esclarecido do referido projeto.

A amostra da presente pesquisa está representada na Tabela 1, sendo composta por 44 crianças de ambos os sexos, com idades entre três anos e cinco meses e oito anos e seis meses, as quais receberam diagnóstico de desvio fonológico, confirmado por meio das avaliações fonoaudiológicas e complementares. Dessas, seis apresentaram desvio fonológico grave, três moderado-grave, 15 leve-moderado e 20 leve.

Todos os sujeitos participantes dos projetos supracitados, inicialmente passaram por uma triagem fonoaudiológica no SAF e, em seguida, foram submetidos às seguintes avaliações fonoaudiológicas: da linguagem compreensiva e expressiva; fonológica; do vocabulário; da articulação; e do sistema estomatognático.

Como avaliação complementar foi realizada avaliação audiológica.

Estas avaliações foram realizadas com o objetivo de confirmar a hipótese de desvio fonológico, portanto os sujeitos participantes da amostra não se encontravam em terapia durante a coleta de dados.

A coleta da amostra de fala das crianças com desvio fonológico (DF) foi realizada utilizando-se a Avaliação Fonológica da Criança ${ }^{(2)}$.

Essa avaliação é realizada através da nomeação e fala espontânea, e possibilita a produção de todos os fones contrastivos em todas as posições que podem ocorrer em relação à estrutura da sílaba e da palavra, obtendo-se uma amostra representativa dos sons da língua ${ }^{(2)}$.

Através da transcrição fonética e da análise contrastiva dos dados de fala, foi determinada a gravidade do desvio fonológico, pelo cálculo do Percentual de Consoantes Corretas $(\mathrm{PCC})^{(16)}$.

O PCC foi obtido mediante a divisão do número de consoantes corretas (NCC) pelo NCC mais o número de consoantes incorretas $(\mathrm{NCI})$, multiplicados por cem $(\mathrm{PCC}=[\mathrm{NCC} /$ $(\mathrm{NCC}+\mathrm{NCI})] \times 100)$.

Conforme os resultados do PCC, os graus de desvio dividem-se em: desvio grave ( $\mathrm{PCC}<50 \%)$, desvio moderado-

* Reed VA. Associations between phonology and other language components in children's communicative performance: clinical implications. Aust $\mathrm{J}$ Hum Comm Dis. $1992 ; 20: 75-87$ 
Tabela 1. Grau de gravidade do desvio fonológico e idade dos sujeitos da amostra

\begin{tabular}{|c|c|c|}
\hline Sujeitos & Grau de gravidade & Idade \\
\hline S1 & Leve & $4 a 1 m$ \\
\hline $\mathrm{S} 2$ & Leve & $4 \mathrm{a} 10 \mathrm{~m}$ \\
\hline S3 & Leve & $6 a 8 m$ \\
\hline S4 & Leve & $6 \mathrm{a} 11 \mathrm{~m}$ \\
\hline S5 & Leve & $6 a 4 m$ \\
\hline S6 & Leve & $7 a 2 m$ \\
\hline S7 & Leve & $7 a 6 m$ \\
\hline S8 & Leve & $6 a 10 m$ \\
\hline S9 & Leve & $8 \mathrm{a} 5 \mathrm{~m}$ \\
\hline $\mathrm{S} 10$ & Leve & $7 a 10 m$ \\
\hline $\mathrm{S} 11$ & Leve & $6 a 10 m$ \\
\hline $\mathrm{S} 12$ & Leve & $5 a 7 m$ \\
\hline $\mathrm{S} 13$ & Leve & $6 a 9 m$ \\
\hline $\mathrm{S} 14$ & Leve & $6 a 7 m$ \\
\hline $\mathrm{S} 15$ & Leve & $5 a 11 m$ \\
\hline $\mathrm{S} 16$ & Leve & $6 a 6 m$ \\
\hline $\mathrm{S} 17$ & Leve & $8 a 2 m$ \\
\hline $\mathrm{S} 18$ & Leve & $5 a 11 m$ \\
\hline S19 & Leve & $6 a 9 m$ \\
\hline $\mathrm{S} 20$ & Leve & $7 a 1 m$ \\
\hline $\mathrm{S} 21$ & Leve-moderado & $6 a 10 m$ \\
\hline $\mathrm{S} 22$ & Leve-moderado & $5 a 4 m$ \\
\hline $\mathrm{S} 23$ & Leve-moderado & $3 a 9 m$ \\
\hline $\mathrm{S} 24$ & Leve-moderado & $6 a 10 m$ \\
\hline $\mathrm{S} 25$ & Leve-moderado & $8 a 2 m$ \\
\hline $\mathrm{S} 26$ & Leve-moderado & $6 a 10 m$ \\
\hline $\mathrm{S} 27$ & Leve-moderado & $5 a 7 m$ \\
\hline $\mathrm{S} 28$ & Leve-moderado & $4 a 7 m$ \\
\hline $\mathrm{S} 29$ & Leve-moderado & $7 a 11 m$ \\
\hline S30 & Leve-moderado & $6 a 5 m$ \\
\hline S31 & Leve-moderado & $4 a 10 m$ \\
\hline S32 & Leve-moderado & $5 a 6 m$ \\
\hline S33 & Leve-moderado & $5 a 2 m$ \\
\hline S34 & Leve-moderado & $6 a 1 m$ \\
\hline S35 & Leve-moderado & $5 a$ \\
\hline S36 & Moderado-grave & $5 a 4 m$ \\
\hline S37 & Moderado-grave & $5 \mathrm{a} 10 \mathrm{~m}$ \\
\hline S38 & Moderado-grave & $6 a 10 m$ \\
\hline S39 & Grave & $3 a 11 m$ \\
\hline S40 & Grave & $5 a 1 m$ \\
\hline S41 & Grave & $3 a 5 m$ \\
\hline S42 & Grave & $4 a$ \\
\hline $\mathrm{S} 43$ & Grave & $7 a 11 m$ \\
\hline S44 & Grave & $4 a 2 m$ \\
\hline
\end{tabular}

Legenda: $\mathrm{a}=$ anos; $\mathrm{m}=$ meses grave ( $\mathrm{PCC}=50$ a $65 \%)$, desvio leve-moderado $(\mathrm{PCC}=65 \mathrm{a}$ $85 \%$ ) e desvio leve ( $\mathrm{PCC}=85$ a $100 \%)$.

Foi realizada, também, a avaliação de vocabulário do teste $\mathrm{ABFW}^{(17)}$ com o objetivo de verificar a competência lexical da criança. Por meio desta prova são avaliados nove campos conceituais: vestuário, animais, alimentos, meios de transporte, móveis e utensílios, profissões, locais, formas e cores, brinquedos e instrumentos musicais. Devem ser apresentadas as figuras correspondentes e solicitar que a criança as nomeie. Essa prova analisa as designações por vocábulos usuais (DVU), as não designações (ND) e os processos de substituição (PS) utilizados pelas crianças para alcançar a nomeação correta dos vocábulos. Para cada um dos campos conceituais há um percentual de respostas que deve ser considerado como adequado a cada faixa etária.

As substituições realizadas pelas crianças em desenvolvimento de linguagem em suas nomeações podem ser classificadas em diferentes tipos de processos de substituição de acordo com as diferenças e semelhanças das unidades lexicais (vocábulos).

Nesta classificação, adotou-se o sistema de categorização dos processos de substituição propostos pelo teste $\mathrm{ABFW}^{(17)}$. Através dos critérios deste teste, classificam-se os processos de substituições de designações em: modificação de categoria gramatical, por exemplo, galo/galinha; substituição por hiperônimo, por exemplo, alface/comida; substituição por co-hipônimo, por exemplo, alface/agrião; substituição por hipônimo, por exemplo, verdura/espinafre; criação de neologismo por analogia morfo-semântico-sintática, por exemplo, chiqueiro/porqueiro; criação de vocábulo foneticamente expressivo, por exemplo, rinoceronte/rinofeçante; substituição por parassinônimo ou equivalente, por exemplo, verde/cor das árvores; substituição por vocábulos que designam seus atributos semânticos, por exemplo, pica-pau/aquele que faz buraquinhos nas árvores; substituição e/ou complementação de semiótica verbal por não verbal, por exemplo, violino/aquele que toca assim (gesto imitando o movimento); substituição e/ ou complementação de semiótica verbal por gesto indicativo, por exemplo, blusa/indica a própria ou a da terapeuta; substituição por paráfrases culturais, por exemplo, canoa/barco de índio; substituição por designação de funções, batedeira/é para fazer bolo; substituição por atributo de co-hipônimo, por exemplo, panela/é para fritar ovo; substituição por paráfrases afetivas, por exemplo, enfermeira/mamãe; valorização do estímulo visual, por exemplo, sanduíche/nomeação de um componente que se destaca na figura; utilização de onomatopeia, por exemplo, cachorro/au-au; e segmento ininteligível, por exemplo, palavra-alvo/ocorre produção oral que não pode ser compreendida.

Para a análise dos dados, primeiramente foi determinado o grau de gravidade do desvio fonológico, através do cálculo do PCC. Após, foi feita a análise dos tipos de processos de substituição mais realizados e dos campos conceituais que os sujeitos apresentaram mais dificuldade na prova de vocabulário do teste $\mathrm{ABFW}^{(17)}$.

Para a verificação dos campos conceituais alterados, foi utilizado o percentual de respostas que deve ser considerado como adequado a cada faixa etária, estando este disponível 
no próprio teste; as faixas etárias utilizadas como referência de normalidade foram de 4:00, 5:00 e 6:00 anos, portanto as faixas etárias anteriores e posteriores terão esses referenciais máximos e mínimos, respectivamente.

Foi realizada análise descritiva para comparar estes resultados entre os diferentes graus de gravidade de desvio fonológico. Devido à diferença significante entre o número de sujeitos em cada grau de gravidade do desvio fonológico, não foi possível utilizar testes estatísticos que mensurassem a significância estatística dos resultados obtidos através do programa SAS (Statistical Analisys System) versão 8.02 , no qual foram estabelecidas tabelas simples e tabelas cruzadas.

\section{RESULTADOS}

A Tabela 2 mostra a porcentagem de crianças dos quatro graus de gravidade do desvio fonológico que realizaram cada um dos processos de substituição.

Devido a não realização de testes que mensurassem a significância estatística, optou-se por considerar as porcentagens médias de cada variável, considerando-se que cada variável é independente, não podendo correlacionar os valores absolutos das tabelas devido às diferenças entre os números de sujeitos de cada grau de gravidade.
Dentre os processos de substituição mais realizados pelas crianças, pôde-se observar que o processo por modificação de categoria gramatical foi igualmente realizado pelas crianças pertencentes aos graus leve e leve-moderado (80\%), seguido do grau moderado-grave $(66,67 \%)$.

O processo de substituição por hiperônimo foi mais encontrado no grau moderado-grave (100\%), sendo ainda elevadas as porcentagens encontradas nos graus leve-moderado $(86,67 \%)$, grave $(83,33 \%)$ e leve $(80 \%)$.

O processo de substituição por co-hipônimo foi igualmente encontrado nos graus leve-moderado, moderado-grave e grave $(100 \%)$, apresentando resultado semelhante no grau leve $(95 \%)$.

O processo de substituição por hipônimo foi mais encontrado no grau moderado-grave (100\%), sendo ainda elevadas as porcentagens encontradas nos graus grave $(66,67 \%)$, leve $(60 \%)$ e leve moderado $(53,33 \%)$.

O processo de substituição por parassinônimo ou equivalente foi mais encontrado no grau moderado-grave (100\%), seguido dos graus leve $(95 \%)$, leve-moderado $(86,67 \%)$ e grave $(83,33 \%)$.

No processo de substituição por vocábulos que designam seus atributos semânticos, apenas o grau leve-moderado apresentou-se com porcentagens elevadas $(53,33 \%)$. Nos demais graus, esse processo não teve porcentagens significantes.

Tabela 2. Porcentagem de utilização de cada processo de substituição utilizado pelos sujeitos com desvios fonológicos nos quatro graus de gravidade

\begin{tabular}{|c|c|c|c|c|c|c|c|c|}
\hline \multirow[t]{2}{*}{ Classificação dos Processos de substituições* } & \multicolumn{2}{|c|}{$\begin{array}{l}\text { Leve } \\
(\mathrm{n}=20)\end{array}$} & \multicolumn{2}{|c|}{$\begin{array}{l}\text { Leve-moderado } \\
\qquad(\mathrm{n}=15)\end{array}$} & \multicolumn{2}{|c|}{$\begin{array}{l}\text { Moderado-grave } \\
\qquad(\mathrm{n}=3)\end{array}$} & \multicolumn{2}{|c|}{$\begin{array}{l}\text { Grave } \\
(n=6)\end{array}$} \\
\hline & $\mathrm{n}$ & $\%$ & $\mathrm{n}$ & $\%$ & $\mathrm{n}$ & $\%$ & $\mathrm{n}$ & $\%$ \\
\hline Modificação de categoria gramatical & 16 & $80 \%$ & 12 & $80 \%$ & 2 & $66,67 \%$ & 3 & $50 \%$ \\
\hline Substituição por hiperônimo & 16 & $80 \%$ & 13 & $86,67 \%$ & 3 & $100 \%$ & 5 & $83,33 \%$ \\
\hline Substituição por co-hipônimo & 19 & $95 \%$ & 15 & $100 \%$ & 3 & $100 \%$ & 6 & $100 \%$ \\
\hline Substituição por hipônimo & 12 & $60 \%$ & 8 & $53,33 \%$ & 3 & $100 \%$ & 4 & $66,67 \%$ \\
\hline Criação de neologismo por analogia morfo-semântico-sintática & 4 & $20 \%$ & 1 & $6,67 \%$ & 0 & $0 \%$ & 0 & $0 \%$ \\
\hline Criação de vocábulo foneticamente expressivo & 0 & $0 \%$ & 2 & $13,33 \%$ & 0 & $0 \%$ & 0 & $0 \%$ \\
\hline Substituição por parassinônimo ou equivalente & 19 & $95 \%$ & 13 & $86,67 \%$ & 3 & $100 \%$ & 5 & $83,33 \%$ \\
\hline $\begin{array}{l}\text { Substituição por vocábulos que designam seus atributos } \\
\text { semânticos }\end{array}$ & 9 & $45 \%$ & 8 & $53,33 \%$ & 1 & $33,33 \%$ & 2 & $33,33 \%$ \\
\hline $\begin{array}{l}\text { Substituição e/ou complementação de semiótica verbal por não- } \\
\text { verbal }\end{array}$ & 0 & $0 \%$ & 0 & $0 \%$ & 0 & $0 \%$ & 1 & $16,67 \%$ \\
\hline $\begin{array}{l}\text { Substituição e/ou complementação de semiótica verbal por gesto } \\
\text { indicativo }\end{array}$ & 0 & $0 \%$ & 0 & $0 \%$ & 0 & $0 \%$ & 0 & $0 \%$ \\
\hline Substituição por paráfrases culturais & 0 & $0 \%$ & 1 & $6,67 \%$ & 0 & $0 \%$ & 1 & $16,67 \%$ \\
\hline Substituição por designação de funções & 12 & $80 \%$ & 10 & $66,67 \%$ & 1 & $33,33 \%$ & 3 & $50 \%$ \\
\hline Substituição por atributo de co-hipônimo & 1 & $5 \%$ & 2 & $13,33 \%$ & 0 & $0 \%$ & 1 & $16,67 \%$ \\
\hline Substituição por paráfrases afetivas & 1 & $5 \%$ & 1 & $6,67 \%$ & 0 & $0 \%$ & 1 & $16,67 \%$ \\
\hline Valorização do estímulo visual & 20 & $100 \%$ & 14 & $93,33 \%$ & 3 & $100 \%$ & 5 & $83,33 \%$ \\
\hline Utilização de onomatopéia & 2 & $10 \%$ & 2 & $13,33 \%$ & 0 & $0 \%$ & 0 & $0 \%$ \\
\hline Segmento ininteligível & 6 & $30 \%$ & 4 & $26,67 \%$ & 1 & $33,33 \%$ & 2 & $33,33 \%$ \\
\hline
\end{tabular}

Legenda: $\mathrm{n}$ refere-se ao número de sujeitos.

*Fonte: Befi-Lopes DM. Vocabulário. In: Andrade CRF, Befi-Lopes DM, Fernandes FDM, Wertzner HF. ABFW: teste de linguagem infantil nas áreas de fonologia, vocabulário, fluência e pragmática. Barueri: Pró-Fono; 2000. cap. 2. 
Tabela 3. Porcentagem de sujeitos com desempenho abaixo do esperado para a faixa etária em cada campo conceitual, em cada grau de gravidade do desvio fonológico

\begin{tabular}{|c|c|c|c|c|c|c|c|c|}
\hline \multirow[t]{2}{*}{ Campos conceituais } & \multicolumn{2}{|c|}{ Leve $(n=20)$} & \multicolumn{2}{|c|}{ Leve-moderado $(n=15)$} & \multicolumn{2}{|c|}{ Moderado-grave $(n=3)$} & \multicolumn{2}{|c|}{ Grave $(n=6)$} \\
\hline & $\mathrm{n}$ & $\%$ & $\mathrm{n}$ & $\%$ & $\mathrm{n}$ & $\%$ & $\mathrm{n}$ & $\%$ \\
\hline Vestuário & 3 & $15 \%$ & 6 & $40 \%$ & 1 & $33,33 \%$ & 1 & $16,67 \%$ \\
\hline Animais & 7 & $35 \%$ & 4 & $26,67 \%$ & 1 & $33,33 \%$ & 0 & $0 \%$ \\
\hline Alimentos & 17 & $85 \%$ & 8 & $53,33 \%$ & 2 & $66,67 \%$ & 5 & $83,33 \%$ \\
\hline Meios de transporte & 0 & $0 \%$ & 2 & $13,33 \%$ & 0 & $0 \%$ & 1 & $16,67 \%$ \\
\hline Móveis e utensílios & 0 & $0 \%$ & 0 & $0 \%$ & 0 & $0 \%$ & 1 & $16,67 \%$ \\
\hline Profissões & 4 & $20 \%$ & 7 & $46,67 \%$ & 0 & $0 \%$ & 1 & $16,67 \%$ \\
\hline Locais & 15 & $75 \%$ & 11 & $73,33 \%$ & 3 & $100 \%$ & 5 & $83,33 \%$ \\
\hline Formas e cores & 7 & $35 \%$ & 6 & $40 \%$ & 0 & $0 \%$ & 1 & $16,67 \%$ \\
\hline Brinquedos e instrumentos musicais & 4 & $20 \%$ & 8 & $53,33 \%$ & 0 & $0 \%$ & 2 & $33,33 \%$ \\
\hline
\end{tabular}

O processo de substituição por designação de funções foi mais encontrado nas crianças pertencentes ao grau leve $(80 \%)$, seguido do grau leve-moderado $(66,67 \%)$.

O processo de substituição por valorização do estímulo visual foi igualmente realizado pelas crianças pertencentes aos graus leve e moderado-grave (100\%), seguido dos graus leve-moderado $(93,33 \%)$ e grave $(83,33 \%)$.

Os demais processos de substituição não obtiveram porcentagens elevadas por parte das crianças dos quatro graus de gravidade estudados.

Observou-se que o processo de substituição predominante na maior parte das crianças, independente do grau de gravidade, foi o processo co-hipônimo, com uma média das porcentagens de $98,75 \%$ entre todas as crianças. Este processo ocorre quando a criança substitui um vocábulo por um termo semanticamente próximo. Além disto, nota-se que as crianças pertencentes ao grau leve-moderado apresentaram maior média entre as porcentagens de processos de substituição $(41,57 \%)$.

A Tabela 3 mostra a porcentagem de crianças dos quatro graus de gravidade do desvio fonológico que apresentaram alterações em cada campo conceitual da prova de vocabulário expressivo.

Foi considerado alterado aquele campo conceitual que apresentou uma média de substituições que não estava de acordo com os parâmetros de normalidade estabelecidos pela prova $^{(17)}$ para cada faixa etária estudada. Após, analisou-se a porcentagem de crianças que apresentaram alterações nos nove campos conceituais da prova, em cada grau de gravidade.

Observou-se, para cada campo conceitual, o grau de gravidade que apresentou a maior média entre as porcentagens de crianças com alteração.

Com esta análise constatou-se que nos campos conceituais "animais" (35\%) e "alimentos" (85\%) há maior porcentagem de crianças do grau leve com alterações.

Nos campos conceituais "vestuário" (40\%), "profissões" (46,67\%), "formas e cores" (40\%) e "brinquedos e instrumentos musicais" $(53,33 \%)$ há maior porcentagem de crianças do grau leve-moderado com alterações.

Já nos campos conceituais "meios de transporte" $(16,67 \%)$ e "móveis e utensílios domésticos" (16,67\%) há maior porcentagem de crianças com alterações no grau grave.
O campo conceitual "locais" (100\%) apresentou maior porcentagem de crianças com alteração no grau moderadograve, sendo este último o campo conceitual que apresentou maior média de porcentagens das crianças com alteração, independente do grau de gravidade $(82,92 \%$ das crianças da amostra apresentaram este campo alterado).

É possível observar também que o grau leve-moderado foi o grau que apresentou a maior média percentual de campos conceituais alterados $(38,52 \%)$, seguido do grau leve $(31,67 \%)$, grave $(31,48 \%)$ e moderado-grave $(25,93 \%)$.

\section{DISCUSSÃO}

Em relação aos tipos de processos de substituição, o mais realizado pelas crianças, independentemente do grau de gravidade do desvio fonológico, foi a substituição por co-hipônimo, que se caracteriza por ser uma substituição por um termo semanticamente próximo. Por exemplo, quando a criança substitui a palavra "alface" pela palavra "cenoura", ou "alface" por "agrião"(17).

As substituições por co-hipônimos, ou seja, erros semanticamente associados são evidências de representações semântico-lexicais difusas ou de que os itens lexicais individuais estão pobremente diferenciados em suas representações semântico-lexicais ${ }^{(18-19)}$. Em casos de Distúrbio Específico de Linguagem a ocorrência de substituições de palavras por outras com atributos semânticos próximos pode indicar que a criança conhece o objeto, porém é incapaz de recuperar a palavra ${ }^{(20)}$ sendo que o mesmo parece ser verdade para crianças com desvios fonológicos conforme constatado na presente pesquisa.

Em concordância com este achado, há um estudo ${ }^{(21)}$ que verificou que as crianças, quando substituem vocábulos, utilizam nomes da mesma categoria semântica da figura-alvo e nomes baseados nas propriedades visuais da figura, levando em conta relações semânticas.

Observou-se que no grau leve-moderado há uma maior porcentagem de crianças que faz uso de processos de substituição, o que discorda de um estudo ${ }^{(19)}$ que refere que em teste de nomeação de figuras, mesmo que o indivíduo apresente um número significante de alterações fonológicas, esta alteração está apenas na representação fonológica de um item lexical, 
sugerindo que o acesso e a representação semântica não estão alterados.

Levando em consideração que o grau leve-moderado, em comparação aos graus moderado-grave e grave, possui menos alterações fonológicas, este achado supracitado não coincide com o esperado para este estudo, pois se acreditava que crianças com repertório fonético pequeno tendessem a ter relativamente poucas palavras em seu vocabulário. Procurando justificar esta hipótese, levamos em consideração que crianças que produzem um número limitado de sons na fala não possuem sons suficientes para diferenciar as palavras entre si. Assim, ao tentar produzir diferentes palavras, não é possível contrastar estes sons, produzindo muitas formas homônimas, dificultando a compreensão de fala, e aparentando ter um vocabulário reduzido ${ }^{(11)}$. Além disto, na nomeação, nomes apropriados devem ser ativados entre as palavras já conhecidas no léxico mental e finalmente deve haver uma organização fonológico-articulatória para que uma resposta específica possa ser executada ${ }^{(22)}$.

Logo, esperávamos que as crianças de maior gravidade de desvio fonológico (grau grave) apresentassem vocabulário mais restrito que as demais.

Além desta evidência, o grau leve-moderado também apresentou maior porcentagem de crianças com campos conceituais alterados. Há pesquisas que vão ao encontro deste achado, como o estudo ${ }^{(23)}$ que propôs que a extensão do distúrbio fonológico apresenta uma correlação negativa, entre o estágio de desenvolvimento fonológico da criança e o tamanho do seu vocabulário.

Outra pesquisa ${ }^{(24)}$ constatou que crianças com alteração de linguagem nomeiam com menos frequência as palavras-alvo do que aquelas em desenvolvimento normal. Além disso, elas utilizam maior número de não designações e de processos de substituição do que aquelas com desenvolvimento normal. Sendo o desvio fonológico uma patologia da linguagem, isso também parece ser verdadeiro pelos resultados aqui encontrados.

Apesar disso, os achados do presente estudo discordam dos encontrados em outras pesquisas. Um estudo ${ }^{(13)}$ refere não ter encontrado diferenças estatisticamente significantes nos três graus de gravidade estudados (leve-moderado, moderado-grave e grave), sendo que as médias de acertos das designações usuais, não-designações e processos de substituição, em todos os campos semânticos avaliados foram semelhantes nos três graus de gravidade.

Além deste, outro estudo ${ }^{(9)}$ relacionou o vocabulário de crianças com alterações fonológicas com o de crianças em desenvolvimento normal da linguagem, e concluiu que existem semelhanças entre eles. Ou seja, alterações no desenvolvimento fonológico não interferiram diretamente na aquisição lexical, uma vez que o desempenho em prova de vocabulário dos sujeitos com alterações fonológicas atingiu os escores esperados para a idade.

Observou-se também que o campo conceitual "locais" foi o que apresentou maior porcentagem de crianças com alteração, independente do grau de gravidade do desvio. Este achado pode ser explicado pelo estudo ${ }^{(25)}$ que constatou que fatores como familiaridade e frequência dos objetos a serem nomeados influenciam no processo de nomeação ativando o acesso ao léxico, pois quando os objetos fazem parte do cotidiano das crianças, eles são nomeados com mais facilidade e acerto.

Em outro estudo ${ }^{(26)}$, realizado com crianças entre 22 e 36 meses, verificou-se resultados semelhantes, nos quais palavras relacionadas à categoria "locais" foram as menos frequentes nas falas destas crianças, pois essa categoria requer maior domínio de conhecimento.

Ao entrar em contato com as figuras do teste de vocabulário expressivo, podemos perceber eram difíceis de serem compreendidas fazendo com que as crianças tivessem dificuldades para denominá-las, principalmente as figuras do campo conceitual "locais". A ambiguidade das figuras, acarretada pela falta de clareza nos detalhes visuais, foi também observada em outro estudo ${ }^{(27)}$ e relacionada ao fato de a nomeação das figuras estudadas diferirem dos nomes propostos.

O estudo acima citado ${ }^{(27)}$ relacionou ainda que a nomeação de figuras pode ser influenciada por outros fatores, entre eles, fatores culturais e de desenvolvimento. Verificou-se que, quando os conceitos das figuras não estão adquiridos, pode ocorrer a atribuição de nomes de objetos conhecidos e que são visualmente semelhantes ao apresentado, ou, quando a criança conhece o objeto, mas não tem a palavra correta para designá-lo, ela procura nomes próximos em seu repertório semântico ${ }^{(27)}$.

Embora neste estudo se tenha observado que crianças com desvio fonológico possuem alterações em seu léxico, há controvérsias entre os autores na relação entre a fonologia e o léxico. Devido a estas controvérsias, sugerimos que sejam realizados mais estudos acerca disto, com uma amostra maior, visando confirmar ou não a existência desta relação, o que influenciará o processo terapêutico de crianças com desvio fonológico.

\section{CONCLUSÃO}

Neste estudo encontrou-se que a gravidade do desvio fonológico que apresenta mais alterações em prova de vocabulário é o leve-moderado. Constatou-se também que o uso de co-hipônimo é o processo de substituição mais realizado e o campo conceitual "locais" é o que apresenta maior dificuldade por parte das crianças de um modo geral. 


\begin{abstract}
Purpose: To verify the most frequent substitution processes and the most altered semantic fields presented by children with phonological disorders on an expressive vocabulary test, establishing a possible correlation of these deficits with the severity of their phonological disorders. Methods: The sample comprised 44 subjects with phonological disorders, with ages ranging from three years and five months to eight years and six months. Data were obtained from databases of ongoing research projects from the Language and Speech Studies Center of the Federal University of Santa Maria. The sample was divided into four groups (severe, moderate-severe, mild-moderate, and mild), according to the severity of the phonological disorder, as determined by the Percentage of Correct Consonants. The Expressive Vocabulary subtest of the Language Test for Young Children ABFW was applied. Subsequently, correlational analyses between substitution processes and semantic fields with severity degree were carried out. Results: The co-hyponym substitution process was the most used, regardless of severity degree. It was also observed that children with mild-moderate phonological disorders presented significantly more substitution processes. A greater number of disordered children had poor performances on the semantic field "places", and children with mild-moderate phonological disorders also presented poor performances in more semantic fields. Conclusions: Children with mild-moderate phonological disorders presented more difficulties on the expressive vocabulary test; the most frequently used substitution process was co-hyponym, and the semantic field "places" was generally more difficult for the subjects.
\end{abstract}

Keywords: Vocabulary; Language tests; Speech disorders; Child; Speech; Child Language

\section{REFERÊNCIAS}

1. Bagetti T, Mota HB, Keske-Soares M. A terapia fonológica no tratamento do retardo simples de linguagem. Fono Atual. 2003;26:4250 .

2. Yavas M, Hernandorena CL, Lamprecht RR. Avaliação fonológica da criança: reeducação e terapia. Porto Alegre: Artes Médicas; 1992.

3. Vieira MG, Mota HB, Keske-Soares M. Relação entre idade, grau de severidade do desvio fonológico e consciência fonológica. Rev Soc Bras Fonoaudiol. 2004;9(3):144-50.

4. Linassi LZ, Keske-Soares M, Mota HB. Habilidades de memória de trabalho e o grau de severidade do desvio fonológico. Pró-Fono. 2005;17(3): 383-92.

5. Wertzner HF, Amaro L, Galea DE. Phonological performance measured by speech severity indices compared with correlated factors. São Paulo Med J. 2007; 125(6): 309-14.

6. Wertzner HF, Papp ACCS, Galea DES. Provas de nomeação e imitação como instrumentos de diagnóstico do transtorno fonológico. Pró-Fono. 2006;18(3):303-12.

7. Wertzner HF, Pagan LO, Galea DES, Papp ACCS. Características fonológicas de crianças com transtorno fonológico com e sem histórico de otite média. Rev Soc Bras Fonoaudiol. 2007;12(1):41-7.

8. Mota HB. Terapia fonoaudiológica para os desvios fonológicos. Rio de Janeiro: Revinter; c2001.

9. Befi-Lopes DM, Gandara JP. Desempenho em prova de vocabulário de crianças com diagnóstico de alteração fonológica. Rev Soc Bras Fonoaudiol. 2002; 7(1):16-22.

10. Bassano D, Maillochon I, Eme E. Developmental changes and variability in the early lexicon: a study of French children's naturalistic productions. J Child Lang. 1998;25(3):493-531.

11. Stoel-Gammon C. Normal and disordered phonology in two-years-olds. Top Lang Disord. 1991;11(4):21-32.

12. Fonseca LF, Wertzner HF. Inteligibilidade de fala, gravidade e tipo de erros característicos do transtorno fonológico. In: XIII Congresso Brasileiro de Fonoaudiologia, 2005, Santos. Rev Soc Bras Fonoaudiol. 2005;10 (n. esp).

13. Pereira LF. Desvio fonológico: desempenho de pré-escolares em tarefas lingüísticas e metalingüísticas nos diferentes graus de gravidade [tese]. São Paulo: Universidade Federal de São Paulo - Escola Paulista de Medicina; 2006

14. Pedroso FS, Rotta NT. Transtornos da linguagem. In: Rotta NT, Ohlweiler
L, Riesgo RS. Transtornos da aprendizagem: abordagem neurobiológica e multidisciplinar. Porto Alegre: Artmed; 2006. p. 131-8.

15. Tonietto L, Parente MAMP, Duvignau K, Gaume B, Bosa CA. Aquisição inicial do léxico verbal e aproximações semânticas em português. Psicol Reflex Crit. 2007;20(1):114-23.

16. Shriberg LD, Kwiatkoviski J. Phonological disorders I: a diagnostic classification system. J Speech Hear Disord. 1982;47(3):226-41.

17. Befi-Lopes DM. Vocabulário. In: Andrade CRF, Befi-Lopes DM, Fernandes FDM, Wertzner HF. ABFW: teste de linguagem infantil nas áreas de fonologia, vocabulário, fluência e pragmática. Barueri: PróFono; 2000. cap. 2.

18. Lahey M, Edwards J. Why do children with specific language impairment name pictures more slowly than their peers? J Speech Hear Res. 1996;39(5):1081-98.

19. Lahey M, Edwards J. Naming errors of children with specific language impairment. J Speech Lang Hear Res. 1999;42(1):195-205.

20. Bastos DA, Befi-Lopes DM, Rodrigues A. Habilidade de organização hierárquica do sistema lexical em crianças com distúrbio específico de linguagem. Rev Soc Bras Fonoaudiol. 2006;11(2):82-9.

21. Cowan N, Hulme C. The development of memory in childhood. London: Psychology Press; 1998.

22. Johnson CJ, Paivio A, Clark JM. Cognitive components of picture naming. Psychol Bull.1996;120(1):113-39.

23. Ingram D. The categorization of phonological impairment. In: Hodson BW,

Edwards ML. Perspectives in applied phonology: perspectives and clinical applications. Maryland: Aspen Publishers; 1997. P.19-41.

24. Befi-Lopes DM, Galea DES. Análise do desenvolvimento lexical em crianças com alterações no desenvolvimento da linguagem. Pró-Fono. 2000;12(2):31-7.

25. Scheuer CI, Stivanin L, Mangilli LD. Nomeação de figuras e a memória em crianças: efeitos fonológicos e semânticos. Pró-Fono. 2004;16(1):4956.

26. Pedromônico MRM, Affonso LA, Sañudo A. Vocabulário expressivo de crianças entre 22 e 36 meses: estudo exploratório. Rev Bras Crescimento Desenvolv Hum. 2002;12(2):13-22.

27. Miranda MC, Pompéia S, Bueno OFA. Um estudo comparativo das normas de um conjunto de 400 figuras entre crianças brasileiras e americanas. Rev Bras Psiquiatr. 2004;26(4):226-33. 